
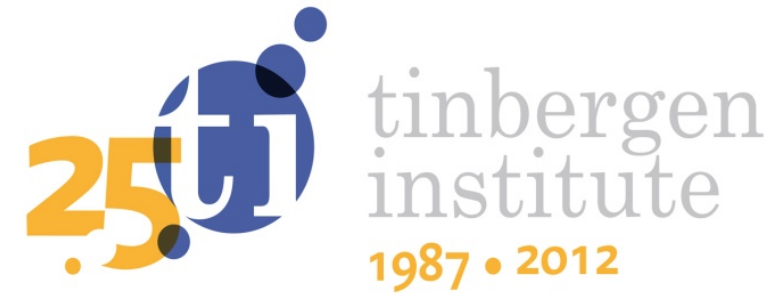

\title{
Leverage and Feedback Effects on Multifactor Wishart Stochastic Volatility for Option Pricing
}

\author{
Manabu Asail \\ Michael McAleer²
}

' Soka University, Japan;

2 Erasmus School of Economics, Erasmus University Rotterdam, Econometric and

Tinbergen Institutes, The Netherlands; Kyoto University, Japan; Complutense University of Madrid, Spain. 
Tinbergen Institute is the graduate school and research institute in economics of Erasmus University Rotterdam, the University of Amsterdam and VU University Amsterdam.

More TI discussion papers can be downloaded at http://www.tinbergen.nl

Tinbergen Institute has two locations:

Tinbergen Institute Amsterdam

Gustav Mahlerplein 117

1082 MS Amsterdam

The Netherlands

Tel.: +31(0)205251600

Tinbergen Institute Rotterdam

Burg. Oudlaan 50

3062 PA Rotterdam

The Netherlands

Tel.: +31(0)10 4088900

Fax: $+31(0) 104089031$

Duisenberg school of finance is a collaboration of the Dutch financial sector and universities, with the ambition to support innovative research and offer top quality academic education in core areas of finance.

DSF research papers can be downloaded at: http://www.dsf.nl/

Duisenberg school of finance

Gustav Mahlerplein 117

1082 MS Amsterdam

The Netherlands

Tel.: +31(0)20 5258579 


\title{
Leverage and Feedback Effects on Multifactor Wishart Stochastic Volatility for Option Pricing*
}

\author{
Manabu Asai \\ Faculty of Economics \\ Soka University, Japan \\ and \\ Wharton School \\ University of Pennsylvania
}

\author{
Michael McAleer \\ Econometric Institute \\ Erasmus School of Economics \\ Erasmus University Rotterdam \\ and \\ Tinbergen Institute \\ The Netherlands \\ and \\ Institute of Economic Research \\ Kyoto University, Japan \\ and \\ Department of Quantitative Economics \\ Complutense University of Madrid, Spain
}

December 2012

${ }^{*}$ The authors are most grateful to Yoshi Baba and Christian Hafner for very helpful comments and suggestions. The first author acknowledges the financial support of the Japan Ministry of Education, Culture, Sports, Science and Technology, Japan Society for the Promotion of Science, and Australian Academy of Science. The second author is most grateful for the financial support of the Australian Research Council, National Science Council, Taiwan, and the Japan Society for the Promotion of Science. Address for correspondence: Faculty of Economics, Soka University, 1-236 Tangi-cho, Hachioji, Tokyo 192-8577, Japan. Email address: m-asai@soka.ac.jp. 


\begin{abstract}
The paper proposes a general asymmetric multifactor Wishart stochastic volatility (AMWSV) diffusion process which accommodates leverage, feedback effects and multifactor for the covariance process. The paper gives the closed-form solution for the conditional and unconditional Laplace transform of the AMWSV models. The paper also suggests estimating the AMWSV model by the generalized method of moments using information not only of stock prices but also of realized volatilities and covolatilities. The empirical results for the bivariate data of the NASDAQ 100 and S\&P 500 indices show that the general AMWSV model is preferred among several nested models.
\end{abstract}

Keywords: Multivariate Stochastic Volatility; Wishart Process; Leverage Effects; Feedback Effects; Multifactor Model; Option Pricing.

JEL classifications: C32, C51, G13 


\section{Introduction}

In the framework of discrete time models, a wide range of multivariate GARCH and stochastic volatility (SV) models has been developed, analyzed, and applied extensively in recent years to characterize the volatility that is inherent in high frequency financial time series data. Bauwens, Laurent and Rombouts (2006) provided a survey of multivariate GARCH models, while Asai, McAleer and Yu (2006) and Chib, Omori and Asai (2009) evaluated various kinds of multivariate SV models.

There has been growing interest in the continuous-time multivariate stochastic volatility processes, especially in (i) estimating integrated volatility and co-volatility, (ii) deriving the diffusion limit of discrete multivariate volatility models, and (iii) developing option pricing models. With respect to the first point, several authors, including Hayashi and Yoshida (2005), Voev and Lunde (2007), Zhang (2008), Malliavin and Mancino (2009), Wang and Zhou (2010) and Barndorff-Nielsen et al. (2011), have developed volatility matrix estimators in the context of realized volatility and co-volatility. Regarding diffusion limits in the univariate class, Nelson (1990) derived SV models as the diffusion limit of the GARCH and exponential GARCH (EGARCH) models. Nelson and Foster (1994), Drost and Werker (1996), Duan (1997) and Fornari and Mele (1997) examined various GARCH-type diffusions. While Nelson (1996) developed the continuous record asymptotic theory for several multivariate GARCH models, Hafner, Laurent and Violante (2010) derived the diffusion limit of the consistent DCC model of Aielli (2006) that modified the DCC model of Engle (2002) in such a way that the unstandardized conditional correlation matrix has martingale difference innovations.

For pricing options, Hull and White (1987), Scott (1987), Melino and Turnbull (1990) and Heston (1993) proposed option pricing SV models, while Duan (1997) suggested the option pricing 
GARCH model. With respect to the multivariate derivative pricing models, Gourieroux (2006) and Gourieroux and Sufana (2010) extended Wishart Autoregressive (WAR) diffusion processes, as originally considered by Bru (1991). Recently, Muhle-Karbe, Pfaffel and Stelzer (2012) developed a multivariate Ornstein-Uhlenbeck-type stochastic volatility model based on a Lévy process.

The first purpose of the paper is to extend the WAR models of Gourieroux (2006) and Gourieroux and Sufana (2010) regarding the asymmetric effects and multifactor for the covariance process. There are two ways to introduce asymmetric effects in continuous SV models: one way is to accommodate feedback effects, while another is to have leverage effects. As Gourieroux and Sufana (2010) suggested a WAR model with feedback effects in log-price processes, this paper considers leverage effects in addition to feedback effects. Turning to multifactors, Chernov et al. (2003) found evidence for the superiority of two-factor specifications over one-factor models for S\&P 500 returns, and showed that introducing additional factors contributes to breaking the link between tail thickness and volatility. In this paper, we develop the new general asymmetric multifactor WAR stochastic volatility models.

The second purpose of the paper is to present a closed-from expression of the conditional Laplace transform of the new model as it is the basis for pricing derivatives, as shown in Duffie, Pan and Singleton (2000). The third purpose of the paper is to suggest estimation of the new models using generalized method of moments (GMM), based on the information not only of prices but also of realized volatility and co-volatilities.

The remainder of the paper is organized as follows. Section 2 proposes the new asymmetric multifactor Wishart stochastic volatility models, and derives the conditional and unconditional Laplace transforms. Section 2 also explains the method to price derivatives using Laplace transforms and the Fourier inversion technique. Section 3 suggests GMM estimation via the information 
on stock prices and realized covariances, and conducts Monte Carlo simulations to investigate the finite sample properties of the estimator. Section 4 gives an empirical example for the pair of NASDAQ 100 and S\&P 500 indices, and Section 5 gives the conclusions.

In the following, $W(A, p)$ denotes the Wishart distribution with the scale matrix $A$ and the degrees-of-freedom parameter $p$. For any symmetric matrix $X, X^{1 / 2}$ is defined by the spectral decomposition of $X$ so that $X^{1 / 2} X^{1 / 2}=X$. For any square matrix $X$, the matrix-exponential operator is defined by $\operatorname{Exp}(X)=\sum_{i=0}^{\infty}(1 / i !) X^{i}$, with $X^{0}=I$.

\section{Asymmetric Multifactor Wishart Stochastic Volatility}

\subsection{The Model}

In this section we develop new continuous-time multivariate stochastic volatility (MSV) models, based on the Wishart Autoregressive (WAR) process, as suggested by Bru (1991) and studied by Gourieroux (2006). The $\operatorname{WAR}(\nu, \Phi, C)$ process is defined by

$$
d W_{t}=\left(\nu C^{\prime} C+W_{t} \Phi^{\prime}+\Phi W_{t}\right) d t+W_{t}^{1 / 2} d B_{t}^{M}\left(C^{\prime} C\right)^{1 / 2}+\left(C^{\prime} C\right)^{1 / 2} d B_{t}^{M} W_{t}^{1 / 2}
$$

where the $B_{t}^{M}$ are $n$ dimensional and symmetric matrix-variate standard Brownian motions, $\nu$ is a scalar such that $\nu>n, \Phi$ is an $n \times n$ matrix, and $C$ is an $n$ dimensional upper triangular matrix.

Instead of specifying $W_{t}$ as an MSV process, we consider a multifactor specification of WAR process as follows. Let $\Omega_{t}$ be an $n$ dimensional square matrix defined by

$$
\begin{aligned}
\Omega_{t} & =\Theta^{\prime}\left(\sum_{k=1}^{K} V_{k t}\right) \Theta, \\
d V_{k t} & =\left(\nu_{k} I_{n}+V_{k t} \Phi_{k}^{\prime}+\Phi_{k} V_{k t}\right) d t+V_{k t}^{1 / 2} d B_{k t}^{M}+d B_{k t}^{M} V_{k t}^{1 / 2},
\end{aligned}
$$

where the $B_{k t}$ 's are symmetric matrix-variate standard Brownian motions, and are mutually independent. In this specification, each $V_{k t}$ follows a $\operatorname{WAR}\left(\nu_{k}, \Phi_{k}, I_{n}\right)$ process independently. In the following, we work with the multifactor specification defined by $\Omega_{t}$. 
Let $p_{t}$ be an $m$ dimensional vector of financial log-prices evolving in continuous time. In addition to MSV, the price process accommodates the feedback and leverage effects as follows. We consider

$$
d p_{t}=\mu^{*}\left(\Omega_{t}\right) d t+d u_{t},
$$

with feedback effect, $\mu^{*}\left(\Omega_{t}\right)$, defined by

$$
\mu^{*}\left(\Omega_{t}\right)=\left(\mu_{1}+\operatorname{tr}\left(M_{1} \Omega_{t}\right), \ldots, \mu_{n}+\operatorname{tr}\left(M_{n} \Omega_{t}\right)\right)^{\prime},
$$

where $\mu_{i}$ and $M_{i}(i=1, \ldots, n)$ are scalar parameters and $n$-dimensional symmetric matrices of parameters, respectively, and the second term in (4) accommodates leverage effects as

$$
d u_{t}=\sum_{k=1}^{K} \Theta^{\prime} V_{k t}^{1 / 2} R_{k} \operatorname{vec}\left(d B_{k t}^{M}\right)+\left(\sum_{k=1}^{K} \Theta^{\prime} V_{k t}^{1 / 2}\left(I_{n}-R_{k} R_{k}^{\prime}\right) V_{k t}^{1 / 2} \Theta\right)^{1 / 2} d B_{t}
$$

where $B_{t}$ is an $n$-vector of standard Brownian motions, and $R_{k}(k=1, \ldots, K)$ are $n \times n^{2}$ matrices of parameters. We assume that $\left(I_{n}-R_{k} R_{k}^{\prime}\right)$ are positive definite matrices. It is straightforward to show that $E\left(d u_{t} d u_{t}^{\prime} \mid \Omega_{t}\right)=\Omega_{t}$. We impose restrictions on $R_{k}$ to reduce the number of parameters, such that $R_{k}=\left(I_{n} \otimes \rho_{k}^{\prime}\right)$, where $\rho_{k}$ is an $n$-vector of parameters. Instead of equation (6), we may also consider a specification given by

$$
d u_{t}=\Omega_{t}^{1 / 2}\left[\sum_{k=1}^{K} R_{k} \operatorname{vec}\left(d B_{k t}^{M}\right)+\left(I_{n}-\sum_{k=1}^{K} R_{k} R_{k}^{\prime}\right) d B_{t}\right] .
$$

However, we will work with (6) for the computational convenience in calculating the Laplace transform. We will refer to the new model in (2)-(6) as the 'general asymmetric multifactor Wishart stochastic volatility' (AMWSV) model.

The new AMWSV model contains seven special cases. If $k=1, R_{k}=O$ and $M_{i}=O$, the AMWSV model reduces to a simple Wishart stochastic volatility (WSV) model with no asymmetric effects. When $k=1$ and $R_{k}=O$, we have a Wishart stochastic volatility model with 
feedback effects (WSV-F). For the case $k=1$ and $M_{i}=O$, we obtain a WSV model with leverage effects (WSV-L). Setting $k=1$ yields a general asymmetric WSV (AWSV) model. Similarly, we have the multifactor WSV model (MWSV), the MWSV model with feedback effects (MWSVF), and the MWSV model with leverage effects (MWSV-L). Note that the model suggested by Gourieroux and Sufana (2010) is essentially the same as the one-factor WSV-F model.

\subsection{Laplace Transforms}

As explained in Duffie, Pan and Singleton (2000), the risk-neutral Laplace transform is the basis for derivative pricing because it can be used to obtain explicit or quasi-explicit prices for various derivatives. In this subsection, we derive the conditional and unconditional Laplace transforms of $p_{t}$ and $\Omega_{t}$. We discuss their risk-neutral Laplace transforms in the next subsection.

Applying the approach of Gourieroux and Sufana (2010), we can show that the joint process $\left(p_{t}, \Omega_{t}\right)$ is an affine process. In order to derive the conditional Laplace transform of $p_{t}$, we use the matrix Riccati linearization technique suggested by Fonseca, Grasselli and Tebaldi (2008), instead the approach of Gourieroux and Sufana (2010). Proposition 1 shows the conditional Laplace transform of the log-price process, $p_{t}$.

Proposition 1 The conditional Laplace transform of the affine process (4) with equations (2), (3), (5) and (6) is

$$
\left.\Psi_{\gamma, t}(h) \equiv E_{t}\left[\exp \left(\gamma^{\prime} p_{t+h}\right\}\right)\right]=\exp \left[\sum_{k=1}^{K} \operatorname{tr}\left(A_{k}(h) V_{k t}\right)+b(h)^{\prime} p_{t}+c(h)\right]
$$

where the symmetric matrices $A_{k}(h)$, vector $b(h)$ and scalar $c(h)$ satisfy the system of Riccati 
equations:

$$
\begin{aligned}
\frac{d}{d h} A_{k}(h) & =\Psi_{k}^{\prime} A_{k}(h)+A_{k}(h) \Psi_{k}+2\left[A_{k}(h)\right]^{2}+2 \Theta\left(\gamma \rho_{k}^{\prime}\right) A_{k}(h)+\Gamma^{*} \quad(k=1, \ldots, n), \\
\frac{d}{d h} b(h) & =0 \\
\frac{d}{d h} c(h) & =\sum_{k=1}^{K} \operatorname{tr}\left(\nu_{k} A_{k}(h)\right)+\gamma^{\prime} \mu
\end{aligned}
$$

with $\Gamma^{*}=\frac{1}{2} \Theta \gamma \gamma^{\prime} \Theta^{\prime}+\sum_{i=1}^{n} \gamma_{i} \Theta M_{i} \Theta^{\prime}, \mu=\left(\mu_{1}, \ldots, \mu_{n}\right)^{\prime}$ and initial conditions $A_{k}(0)=O, b(0)=\gamma$ and $c(0)=0$. Their solutions are given by

$$
\begin{aligned}
A_{k}(h) & =\left[N_{22, k}(h)\right]^{-1} N_{21, k}(h), \quad(k=1, \ldots, K), \\
b(h) & =\gamma, \\
c(h) & =-\frac{1}{2} \sum_{k=1}^{K} \nu_{k}\left[\log \operatorname{det}\left(N_{22, k}(h)\right)+h \operatorname{tr}\left(\Psi_{k}^{\prime}+2 \Theta \gamma \rho_{k}\right)\right],
\end{aligned}
$$

where

$$
\left(\begin{array}{cc}
N_{11, k}(h) & N_{12, k}(h) \\
N_{21, k}(h) & N_{22, k}(h)
\end{array}\right)=\operatorname{Exp} h\left(\begin{array}{cc}
\Psi_{k} & -2 I_{n} \\
\Gamma^{*} & -\left(\Psi_{k}^{\prime}+2 \Theta \gamma \rho_{k}^{\prime}\right)
\end{array}\right)
$$

if $\left[N_{22, k}(h)\right]^{-1}$ exists.

Proof. See Appendix A.1.

Proposition 2 shows the conditional Laplace transform of the multifactor WAR process, $\Omega_{t}$.

Proposition 2 The conditional Laplace transform of the multifactor WAR process (2) with equation (3) is

$$
\begin{aligned}
\Psi_{\Xi, t}^{\dagger}(h) & \equiv E_{t}\left[\exp \left(\operatorname{tr}\left\{\Xi \Omega_{t+h}\right\}\right)\right] \\
& =\prod_{k=1}^{K} \frac{\exp \left(\operatorname{tr}\left\{\left(I_{n}-2 \Theta \Xi \Theta^{\prime} \Sigma_{k}(h)\right)^{-1} \Theta \Xi \Theta^{\prime} P_{k}(h) V_{k t} P_{k}(h)^{\prime}\right\}\right)}{\left[\operatorname{det}\left(I_{n}-2 \Theta \Xi \Theta^{\prime} \Sigma_{k}(h)\right)\right]^{\nu_{k} / 2}},
\end{aligned}
$$

where $\Xi$ is a square matrix,

$$
\begin{aligned}
P_{k}(h) & =\operatorname{Exp}\left(\Phi_{k} h\right), \\
\operatorname{vec}\left(\Sigma_{k}(h)\right) & =\left[\left(I_{n} \otimes \Phi_{k}\right)+\left(\Phi_{k} \otimes I_{n}\right)\right]^{-1} \operatorname{vec}\left(\operatorname{Exp}\left(\Phi_{k} h\right) \operatorname{Exp}\left(\Phi_{k}^{\prime} h\right)-I_{n}\right),
\end{aligned}
$$


if $\Phi_{k}^{-1}$ exists.

Proof. See Appendix A.2.

Applying the same arguments to the original WAR process, we have a similar result.

Corollary 1 The conditional Laplace transform of the WAR( $(\Phi, C)$ process (1) is

$$
\Psi_{\Xi, t}^{*}(h) \equiv E_{t}\left[\exp \left(\operatorname{tr}\left\{\Xi W_{t+h}\right\}\right)\right]=\frac{\exp \left(\operatorname{tr}\left\{\left(I_{n}-2 \Xi \Sigma(h)\right)^{-1} \Xi P(h) W_{t} P(h)^{\prime}\right\}\right)}{\left[\operatorname{det}\left(I_{n}-2 \Sigma(h) \Xi\right)\right]^{\nu / 2}},
$$

where $\Xi$ is a square matrix,

$$
\begin{aligned}
P(h) & =\operatorname{Exp}(\Psi h), \\
\operatorname{vec}(\Sigma(h)) & =\left[\left(I_{n} \otimes \Phi\right)+\left(\Phi \otimes I_{n}\right)\right]^{-1} \operatorname{vec}\left(\operatorname{Exp}(\Phi h) C^{\prime} C \operatorname{Exp}\left(\Phi^{\prime} h\right)-C^{\prime} C\right),
\end{aligned}
$$

if $\Phi^{-1}$ exists. Furthermore, an alternative form of $\Sigma(h)$ is given by

$$
\Sigma(h)=\int_{0}^{h} \operatorname{Exp}\left(\Phi_{k} s\right) C^{\prime} C \operatorname{Exp}\left(\Phi_{k}^{\prime} s\right) d s
$$

Proof. See Appendix A.3.

With respect to Corollary 1, Proposition 5 of Gourieroux (2006) also showed the conditional Laplace transform (12) based on (14), using a different approach. However, Corollary 1 shows that we can obtain $\Sigma(h)$ without any numerical integration.

By setting $h \rightarrow \infty$, we have the unconditional Laplace transform of the WAR process, as in the following corollary.

Corollary 2 The unconditional Laplace transform of the WAR( $\nu, \Phi, C)$ process (1) is

$$
E\left[\exp \left(\operatorname{tr}\left\{\Xi W_{t+h}\right\}\right)\right]=\lim _{h \rightarrow \infty} \Psi_{\Xi, t}^{*}(h)=\left[\operatorname{det}\left(I_{n}-2 \Sigma(\infty) \Xi\right)\right]^{-\nu / 2},
$$

where $\Xi$ is a square matrix and

$$
\operatorname{vec}(\Sigma(\infty))=-\left[\left(I_{n} \otimes \Phi\right)+\left(\Phi \otimes I_{n}\right)\right]^{-1} \operatorname{vec}\left(C^{\prime} C\right),
$$


if all eigenvalues of $\Phi$ are negative. In other words, if $W_{t} \sim W A R(\nu, \Phi, C)$, the invariant distribution of $W_{t}$ is the Wishart distribution with the scale matrix $\Sigma(\infty)$ and the degrees-of-freedom parameter $\nu$.

Proof. If all the eigenvalues of $\Phi$ are negative, $P(h)=\operatorname{Exp}(\Psi h) \rightarrow O$ as $h \rightarrow \infty$, by the spectral decomposition shown in Appendix A.3. The invariant distribution, which is characterized by the moment generating function (16), is the Wishart distribution.

Define the vector of asset returns as $y_{t+h}=p_{t+h}-p_{t}$. The next corollary shows the moment generating functions of $y_{t+h}$ and $\Omega_{t+h}$, which will be used for estimating the AMWSV models.

Corollary 3 For the AMWSV process (2)-(6), the moment generating functions of $y_{t+h}$ and $\Omega_{t+h}$ are given by

$$
\begin{aligned}
E\left[\exp \left(\gamma^{\prime} y_{t+h}\right)\right] & =\exp (c(h)) \prod_{k=1}^{K}\left[I_{n}-2 A_{k}(h) \Sigma_{k}(\infty)\right]^{\nu_{k} / 2} \\
E\left[\exp \left(\operatorname{tr}\left\{\Xi \Omega_{t+h}\right\}\right)\right] & =\prod_{k=1}^{K}\left[I_{n}-2 \Theta \Xi \Theta^{\prime} \Sigma_{k}(\infty)\right]^{\nu_{k} / 2},
\end{aligned}
$$

where $A_{k}(h)$ and $c(h)$ are defined by Proposition 1, and

$$
\operatorname{vec}\left(\Sigma_{k}(\infty)\right)=-\left[\left(I_{n} \otimes \Phi_{k}\right)+\left(\Phi_{k} \otimes I_{n}\right)\right]^{-1} \operatorname{vec}\left(I_{n}\right)
$$

Proof. By a simple application of Corollary 2 to equation (10), we have equation (18). For each $V_{k t}$, we also have $E\left[\exp \left(\operatorname{tr}\left\{\Xi V_{k, t+h}\right\}\right)\right]=\left[I_{n}-2 \Xi \Sigma_{k}(\infty)\right]^{\nu_{k} / 2}$ from Corollary 2. Then we obtain

$$
\begin{aligned}
E\left[\exp \left(\gamma^{\prime}\left(p_{t+h}-p_{t}\right)\right)\right] & =E E_{t}\left[\exp \left(\gamma^{\prime}\left(p_{t+h}-p_{t}\right)\right)\right] \\
& =E \exp \left(-\gamma^{\prime} p_{t}\right) \Psi_{\gamma, t}(h) \\
& =\exp (c(h)) \exp \left[\sum_{k=1}^{K} \operatorname{tr}\left(A_{k}(h) V_{k t}\right)\right] \\
& =\exp (c(h)) \prod_{k=1}^{K}\left[I_{n}-2 A_{k}(h) \Sigma_{k}(\infty)\right]^{\nu_{k} / 2}
\end{aligned}
$$




\subsection{Option Pricing}

Using the result of Duffie, Pan and Singleton (2000), we can use the risk-neutral Laplace transform to solve various option pricing problems. Under the risk-neutral measure $Q$, we want to determine the price $E^{Q}\left(e^{-r T} f\left(p_{T}\right)\right)$ of a European option with payoff $f\left(p_{T}\right)$ at maturity $T$, where $r$ is an instantaneous interest rate and $f$ is a measurable function such that $f: \Re^{n} \rightarrow \Re_{+}$.

In order to obtain the risk-neutral Laplace transform, we can apply the results of Proposition 6 derived by Gourieroux and Sufana (2010) to our case. We need to adjust the drift terms of $\left(p_{t}, \Omega_{t}\right)$ under the risk-neutral distribution, such that

$$
\begin{aligned}
E_{t}^{Q}\left(d p_{t}\right) & =r \iota-\frac{1}{2}\left(e_{1}^{\prime} \Omega_{t} e_{1}, \ldots, e_{n}^{\prime} \Omega_{t} e_{n}\right)^{\prime} d t \\
E_{t}^{Q}\left(d \Omega_{t}\right) & =E_{t}\left(d \Omega_{t}\right)+\operatorname{Cov}\left[\operatorname{tr}\left(C_{t} d \Omega_{t}\right), d \Omega_{t}\right] \\
& =E_{t}\left(d \Omega_{t}\right)+2\left(\Omega_{t} \Xi_{t} \Theta^{\prime} \Theta+\Theta^{\prime} \Theta \Xi_{t}^{\prime} \Omega_{t}\right) d t
\end{aligned}
$$

where $E_{t}^{Q}$ denotes the conditional expectation under the risk-neutral measure $Q, \iota$ is an $n$-vector of ones, and $e_{i}$ is the canonical vector with zero components, except for the $i$ th element which is equal to one. The risk premium on the Brownian motion of the return equation is fixed by the martingale condition, whereas the risk premia corresponding to the covariance can be fixed arbitrarily. As in Gourieroux and Sufana (2010), we consider the case that the volatility risk premia are constant, such that $\Xi_{t}=\bar{\Xi}$. Then we may have the alternative form the above equations:

$$
\begin{aligned}
E_{t}^{Q}\left(d p_{t}\right) & =\left(\bar{\mu}_{1}+\operatorname{tr}\left(\bar{M}_{1} \Omega_{t}\right), \ldots, \bar{\mu}_{n}+\operatorname{tr}\left(\bar{M}_{n} \Omega_{t}\right)\right)^{\prime} d t \\
E_{t}^{Q}\left(d \Omega_{t}\right) & =E_{t}\left(d \Omega_{t}\right)+\operatorname{Cov}\left[\operatorname{tr}\left(C_{t} d \Omega_{t}\right), d \Omega_{t}\right] \\
& =\sum_{k=1}^{K} \Theta^{\prime}\left(\nu_{k} I_{n}+V_{k t} \bar{\Phi}_{k}^{\prime}+\bar{\Phi}_{k} V_{k t}\right) \Theta d t
\end{aligned}
$$


where $\bar{\mu}_{i}=r, \bar{M}_{i}=-\frac{1}{2} e_{i} e_{i}^{\prime}$ and $\bar{\Phi}_{k}=\Phi+2 \Theta \bar{\Xi} \Theta^{\prime}$. We can obtain the risk-neutral conditional Laplace transform by replacing $E_{t}$ with $E_{t}^{Q}$ in Propositions 1 and 2 . We can also have the risk-neutral conditional Laplace transform of the pair $\left(p_{t}, \Omega_{t}\right)$ by applying the same approach. Compared with the MWSV-F model, the option pricing AMWSV model can accommodate the asymmetric effects flexibly through the leverage effects.

For purposes of calculating option prices, we can explain the approach based on the Fourier inversion technique introduced by Carr and Madan (1999) and extended by Eberlein, Glau and Papapantoleon (2010) to the multivariate case. Let $\hat{f}$ be the Fourier transform of $f$. Eberlein, Glau and Papapantoleon (2010) showed that

$$
E^{Q}\left(e^{-r T} f\left(p_{T}\right)\right)=\frac{e^{-z^{\prime} p_{0}-r T}}{(2 \pi)^{n}} \int_{\Re^{n}} e^{-i u^{\prime} p_{0}} \Psi_{i z-u, 0}(T) \hat{f}(i z-u) d u
$$

where $\Psi_{\gamma, t}(h)$ is the conditional Laplace transform of $p_{t+h}$, and the vector $z$ has to lie in the intersection of the domain of $\Psi$ and $\hat{f}$.

Muhle-Karbe, Pfaffel and Stelzer (2012) give the following formulae for basket and spread options. The payoff function of a basket put option is defined by $f(p)=\left(K-\sum_{j=1}^{n} e^{p_{j}}\right)^{+}, K>0$, and its Fourier transform is given by

$$
\hat{f}(u)=K^{1+i \sum_{j=1}^{n} u_{j}} \frac{\prod_{j=1}^{n} \Gamma\left(i u_{j}\right)}{\Gamma\left(2+i \sum_{j=1}^{n} u_{j}\right)},
$$

for all $u \in \mathcal{C}^{n}$ with $\operatorname{Im}\left(u_{j}\right)<0(j=1, \ldots, n)$. We can derive the price of the corresponding call option using put-call-parity $(K-x)^{+}=(x-K)^{+}-x+K$. With respect to a spread call option, the payoff function is defined by $f(p)=\left(e^{p_{1}}-e^{p_{2}}-K\right)^{+}, K>0$, and its Fourier transform is given by

$$
\hat{f}(u)=\frac{K^{1+i u_{1}+i u_{2}}}{i z_{1}\left(1+i z_{1}\right)} \frac{\Gamma\left(i z_{2}\right) \Gamma\left(-i z_{1}-i z_{2}-1\right)}{\Gamma\left(-i z_{1}-1\right)},
$$


for all $u \in \mathcal{C}^{2}$, with $\operatorname{Im}\left(u_{1}\right)>1, \operatorname{Im}\left(u_{2}\right)<0$ and $\operatorname{Im}\left(u_{1}+u_{2}\right)>1$. We can use Fast Fourier Transform (FFT) methods to compute these prices.

\section{Estimation}

For estimating the AMWSV models, we use a generalized method of moments (GMM) based on the moment generating function of $y_{t+h}$ and $\Omega_{t+h}$. With respect to the one factor WSV-F model with $\Theta=\theta I_{n}$, Gourieroux and Sufana (2010) also use GMM, and their Monte Carlo simulation results indicate that it is hard to estimate $\theta$, even for $T=5000$. In order to cope with the problem, we suggest estimation using realized volatilities and co-volatilities. By the methods of Voev and Lunde (2007), Malliavin and Mancino (2009), Wang and Zhou (2010), Barndorff-Nielsen et al. (2011), and Zhang (2011), we can obtain a consistent estimator of $\Omega_{t}$ (without assuming its structure).

We may call the difference between a consistent estimator of $\Omega_{t}$ and $\Omega_{t}$ itself as the 'realized covariance error'. In univariate realized volatility models, Barndorff-Nielsen and Shephard (2002), Bollerslev and Zhou (2002), and Asai, McAleer, and Medeiros (2012a,b) showed it is useful to use an ad hoc approach that accommodates an error term with constant variance. Here, we consider a Wishart-like disturbance.

Consider a Wishart distribution, $W_{t}^{*} \sim W\left(\left(1 / \nu_{*}\right) \Omega_{t}, \nu_{*}\right)$, with the mean and variance of $\operatorname{vec}\left(W_{t}^{*}\right)$ given by $E\left(\operatorname{vec}\left(\Omega_{t}\right)\right)$ and $E\left(\left(1 / \nu_{*}\right)\left(I_{n}+P\right)\left(\Omega_{t} \otimes \Omega_{t}\right)\right)$, respectively, where $P$ is a matrix which satisfies $\operatorname{vec}\left(A^{\prime}\right)=P \operatorname{vec}(A)$ for any square matrix $A$. Let $X_{t}$ be a consistent estimator of $\Omega_{t}$. We assume that the first two moments of $x_{t}=\operatorname{vec}\left(X_{t}\right)$ correspond to the moments of $\operatorname{vec}\left(W_{t}^{*}\right)$. Although this specification is ad hoc, this structure can create disturbances of $\Omega_{t}$ with a single nuisance parameter, $\nu_{*}$. By construction, we need to guarantee the consistency and positive semi-definiteness of $X_{t}$. We will use the moments of $x_{i j, t}(1 \leq i \leq j \leq n)$ and $x_{i j, t} x_{k l, t}$ 
$(1 \leq i \leq j \leq n)(1 \leq k \leq l \leq n)$, resulting in $n^{*}\left(n^{*}+3\right) / 2$ conditions for $x_{t}$, where $n^{*}=n(n+1) / 2$.

Following Gourieroux and Sufana (2010), we work with the moments of $\exp \left(u_{2}^{\prime} y_{t+2 h}+u_{1}^{\prime} y_{t+h}\right)$ for $y_{t}$. For the case $n=2$, we consider 23 moment conditions of $\left(u_{2}^{\prime}, u_{1}^{\prime}\right):(0,0,1,0),(0,0,0,1)$, $(0,0,2,0),(0,0,0,2),(0,0,1,1),(1,0,1,0),(0,1,0,1),(1,0,0,1),(0,1,1,0),(1,0,2,0),(0,1,0,2)$, $(1,0,0,2),(0,1,2,0),(2,0,2,0),(0,2,0,2),(2,0,0,2),(0,2,2,0),(0,0,3,0),(0,0,0,3),(0,0,3,3)$, $(0,0,4,0),(0,0,0,4),(0,0,4,4)$.

For $n=2$, we use GMM with these 32 moment conditions to estimate 26 parameters of the AMWSV model. We estimate the weighting matrix according to Newey and West (1987), with a lag length of 50 .

We present the results of a Monte Carlo study to investigate the finite sample performance of GMM estimation for $n=2$. We generate $R$ simulated time series of $\left\{y_{t}, x_{t}\right\}_{t=1}^{T}$ for the AMWSV model in equations (2)-(6), and for some given "true" parameter vector. Subsequently, we treat the parameter vector as unknown and estimate it for each series using GMM. We compute the sample mean, standard deviation, and root mean squared error (RMSE), and compare these with the true parameter value. In order to obtain the simulated path of $V_{k t}$, we use the following approach. Noting that $d V_{k t}=V_{k t}^{1 / 2} d V_{k t}^{1 / 2}+\left(d V_{k t}^{1 / 2}\right) V_{k t}^{1 / 2}$, we have

$$
\operatorname{vec}\left(d V_{k t}^{1 / 2}\right)=\left[\left(V_{k t}^{1 / 2} \otimes I_{n}\right)+\left(I_{n} \otimes V_{k t}^{1 / 2}\right)\right]^{-1} \operatorname{vec}\left(\nu I_{n}+V_{k t} \Phi_{k}^{\prime}+\Phi_{k} V_{k t}\right) d t+\operatorname{vec}\left(d B_{k t}^{M}\right),
$$

where $B_{k t}^{M}$ is the same symmetric matrix-variate Brownian motion process used in equation (3). Then we can apply the local linearization technique of Shoji and Ozaki (1998) to generate $V_{k t}^{1 / 2}$ $\left(V_{k t}\right.$ and $\left.\Omega_{t}\right)$ efficiently.

The true parameter values for generating Monte Carlo samples are given in the first column of Table 1, which is obtained from our empirical analysis in Section 4. The results given in Table 1 are for sample size $T=2500$, with the number of iterations set to $R=2000$. Table 1 shows that 
most values of the standard deviations are close to their RMSE counterparts, indicating that the biases in finite samples are negligible.

\section{Empirical Analysis}

The empirical analysis focuses on the pair of indexes given by NASDAQ 100 and S\&P 500. In calculating daily realized volatilities and co-volatilities, we use the averaging realized volatility matrix estimator suggested by Wang and Zou (2010). We clean the data for outliers and discard transactions outside trading hours, considering transactions between 9.30 A.M. and 4.00 P.M. Following Tao et al. (2011), we use grids selected in accord with 5 minute returns to take averages. The sample period is July 1, 1996 to September 28, 2012, giving $T=3961$ observations for the return vector, $y_{t}$, and realized covariance, $X_{t}$.

We estimate one-factor and two-factor WSV models including the simple WSV, WSV-F, WSVL and general AWSV models. For purposes of a diagnostic statistic, we use the conventional GMM J-test of Hansen (1982). Under standard regularity conditions, the minimized value of the objective function multiplied by the sample size is asymptotically distributed as chi-square, which allows for an omnibus test of the overidentifying restrictions. We also examine the estimates of $E\left(\operatorname{tr}\left(M_{i} \Omega_{t}\right)\right)$ and $E\left(d \omega_{i i, t} d u_{1 t}\right)+E\left(d \omega_{i i, t} d u_{2 t}\right) \quad(i=1,2)$ to check the feedback and leverage effects, respectively. Note that $E\left[\operatorname{vec}\left(d \Omega_{t}\right) d u_{t}^{\prime} \mid V_{1 t}, V_{2 t}\right]=\left(\Theta^{\prime} \otimes \Theta^{\prime}\right) \sum_{k=1}^{2}\left[\left(I_{n} \otimes V_{k t}^{1 / 2}\right)+\right.$ $\left.\left(V_{k t}^{1 / 2} \otimes I_{n}\right)\right] R_{k}^{\prime} V_{k t}^{1 / 2} \Theta d t$. We expect $E\left(\operatorname{tr}\left(M_{i} \Omega_{t}\right)\right)>0$ for the feedback effect, while we expect $E\left(d \omega_{i i, t} d u_{1 t}\right)+E\left(d \omega_{i i, t} d u_{2 t}\right)<0$ for the leverage effect.

Table 2 shows the estimates of the one-factor WSV models. For estimating the one factor models, we excluded the last 6 moment conditions for $y_{t}$. With respect to the simple one-factor WSV model, the estimate of $\nu_{1}$ is 17.0 , which is significant at the $5 \%$ level. The estimates of $\phi_{11,1}$ and $\phi_{22,1}$ are negative and significant, while those of $\phi_{12,1}$ and $\phi_{21,1}$ are insignificant. The 
eigenvalues of the estimate of $\Phi_{1}$ are negative, indicating that the invariant distribution of $V_{1 t}$ exists by Corollary 2. The estimate of $\nu_{*}$ is 0.17 and is significant. We can interpret the variance of $x_{t}$ conditional on $\Omega_{t}$, that is, $\left(1 / \nu_{*}\right)\left(I_{n}+P\right)\left(\Omega_{t} \otimes \Omega_{t}\right)$, such that, as $\nu$ decreases, the variance of $x_{t}$ increases. The GMM omnibus test for the overidentifying restrictions rejects the simple one-factor WSV model.

Table 2 also shows the estimates of the one-factor WSV-F, WSV-L and Asymmetric WSV models. Compared with the simple WSV model, the estimates of $\nu_{1}$ became smaller and are close to 2.1 for all three models. The estimates of $\phi_{11,1}$ and $\phi_{22,1}$ are negative and significant, while those of $\phi_{12,1}$ and $\phi_{21,1}$ are insignificant. The estimate of $\nu_{*}$ is close to 0.3 , which is greater than the value for the simple WSV model. With respect to asymmetry, Table 3 summarizes the estimated asymmetric effects. For the WSV-F model, the feedback effect to NASDAQ returns is positive, while the effect to $\mathrm{S} \& \mathrm{P}$ returns is negative. Hence, the specification (especially for the second variable) has room to improve.

Regarding the WSV-L model, the leverage effects can be examined by the estimates of $E\left(d \omega_{i i, t} d u_{1 t}\right)+$ $E\left(d \omega_{i i, t} d u_{2 t}\right)$, as noted above. The leverage effects to the NASDAQ returns volatility is negative, while the effect to the $\mathrm{S} \& \mathrm{P}$ returns volatility is positive. Again, the sign conditions are not satisfied. For the general AWSV model, the feedback effects are positive, while the leverage effects are negative for NASDAQ and S\&P. This time the conditions for asymmetric effects are satisfied. However, the GMM omnibus tests for the overidentifying restrictions reject the WSV-F, WSV-L and AWSV models.

Table 4 reports the estimates of the two-factor WSV models. With respect to the simple two-factor WSV model without asymmetric effects, all the estimated parameters are significant. The estimate of $\nu_{1}$ is 5.8 , while the estimate of $\nu_{2}$ is 5.8. The estimates of $\phi_{11, i}$ and $\phi_{22, i}$ are 
negative for $i=1,2$, while those of $\phi_{12, i}$ and $\phi_{21, i}$ are positive. The estimate of $\nu_{*}$ is 1.5 , which is greater than the values given in Table 2. This result suggests that the unexplained component of $x_{t}$ has become smaller than those of the one-factor models. The GMM omnibus test for the overidentifying restrictions does not reject the simple two-factor WSV model. Except for the parameters of asymmetric effects, all the estimates are significant for the two-factor WSV-F, WSV-L and AWSV models. Compared to the estimates of the simple two-factor WSV model, the WSV-F model has similar results. The WSV-L and AWSV models have smaller $\nu_{*}$ values compared with the simple WSV model. The GMM omnibus tests for the overidentifying restrictions do not reject these three asymmetric two-factor WSV models.

Table 5 presents the estimated asymmetric effects. For the WSV-F model, the feedback effects to NASDAQ and S\&P returns are positive, indicating that the sign condition is satisfied. Regarding the WSV-L model, the leverage effects to the NASDAQ and S\&P returns volatilities are negative. For the general AWSV model, the feedback effects are positive, while the leverage effects are negative for NASDAQ and S\&P. The results also show that the conditions are satisfied.

These empirical results show that the data favor the two-factor WSV models. Among the two-factor WSV models, the general asymmetric specification is preferred by the $t$ tests. The two-factor AWSV model has another merit in that its option pricing variation can accommodate asymmetric effects flexibly via the leverage effect.

\section{Concluding Remarks}

In this paper, we suggested a general asymmetric multifactor Wishart stochastic volatility (AMWSV) model which accommodates leverage and feedback effects and multifactor for the covariance process. We also derived the closed-form expression of the conditional and unconditional Laplace transform of the AMWSV models, the results of which can be used for pricing derivatives. Fur- 
thermore, we proposed to estimate the AMWSV model using GMM based on information not only of stock prices but also of realized volatilities and co-volatilities. The empirical results for the pair of NASDAQ 100 and S\&P 500 indices show the usefulness of the new specification compared with other specifications. 


\section{Appendix}

\section{A.1 Proof of Proposition 1}

By applying the Feynman-Kac argument, we have

$$
\frac{d}{d h} \Psi_{\gamma, t}(h)=\mathcal{L}_{p, V_{1}, \ldots, V_{k}} \Psi_{\gamma, t}(h), \quad \Psi_{\gamma, t}(0)=\exp \left(\gamma^{\prime} p_{t}\right)
$$

with the infinitesimal generator for $\left(p_{t}, V_{1 t}, \ldots, V_{k t}\right)$. By equation (5.12) of Bru (1991), we can obtain the infinitesimal generator for the WAR process, $V_{k t}$, as follows:

$$
\mathcal{L}_{V_{k}}=\operatorname{tr}\left[\left(\nu_{k} I_{n}+V_{k} \Phi_{k}^{\prime}+\Phi_{k} V_{k}\right) D_{k}+2 V_{k} D_{k}^{2}\right]
$$

where $D_{k}$ is a matrix differential operator with elements $D_{i j, k}=\left(\partial / \partial V_{i j, k}\right)$. We define $\delta=$ $\left(\delta_{1}, \ldots, \delta_{n}\right)^{\prime}$ with $\delta_{i}=\left(\partial / \partial p_{i}\right)$. Then the infinitesimal generator for $\left(p_{t}, V_{1 t}, \ldots, V_{k t}\right)$ is given by

$$
\begin{aligned}
\mathcal{L}_{p, V_{1}, \ldots, V_{k}}=\delta^{\prime} \mu & +\sum_{i=1}^{n} \delta_{i} \operatorname{tr}\left[M_{i} \Theta^{\prime}\left(\sum_{k=1}^{K} V_{k}\right) \Theta\right]+\frac{1}{2} \operatorname{tr}\left[\Theta^{\prime}\left(\sum_{k=1}^{K} V_{k}\right) \Theta \delta \delta^{\prime}\right] \\
& +\sum_{k=1}^{K} \operatorname{tr}\left[\left(\nu_{k} I_{n}+V_{k} \Phi_{k}^{\prime}+\Phi_{k} V_{k}\right) D_{k}+2 V_{k} D_{k}^{2}\right]+2 \sum_{k=1}^{K} \operatorname{tr}\left[V_{k} \Theta \gamma \rho_{k} D_{k}\right] .
\end{aligned}
$$

Thus, we obtain

$$
\begin{aligned}
0=- & \sum_{k=1}^{K} \operatorname{tr}\left[\frac{d}{d h} A_{k}(h) V_{k}\right]-\frac{d}{d h} b(h)^{\prime} p-\frac{d}{d h} c(h) \\
& +\sum_{k=1}^{K} \operatorname{tr}\left[\left(\nu_{k} I_{n}+V_{k} \Phi_{k}^{\prime}+\Phi_{k} V_{k}\right) A_{k}(h)+2 V_{k} A_{k}^{2}(h)+V_{k} \Theta \gamma \rho_{k} A_{k}(h)\right] \\
& +\mu^{\prime} b(h)+\sum_{i=1}^{n} b_{i}(h) \operatorname{tr}\left[\Theta M_{i} \Theta^{\prime} \sum_{k=1}^{K} V_{k}\right]+\frac{1}{2} \sum_{k=1}^{K} \operatorname{tr}\left[V_{k} \Theta b(h) b(h)^{\prime} \Theta^{\prime}\right]
\end{aligned}
$$

with boundary conditions $A_{k}(0)=O$ for all $k, b(0)=\gamma$ and $c(0)=0$. By identifying the coefficients of $\left(p, V_{1}, \ldots, V_{k}\right)$, we have the ordinary differential equations for $b(h)$ and $A_{k}(h)$ given by equation (8). As usual, the function $c(h)$ can be obtained by direct integration. 
Following Fonseca, Grasselli and Tebaldi (2008), we linearize the Matrix Riccati equation for $A_{k}(h)$ by doubling the dimension of the problem. Consider a decomposition which satisfies

$$
A_{k}(h)=\left[F_{k}(h)\right]^{-1} G_{k}(h) .
$$

Then we have

$$
\frac{d}{d h}\left[F_{k}(h) A_{k}(h)\right]-\frac{d}{d h}\left[F_{k}(h)\right] A_{k}(h)=F_{k}(h) \frac{d}{d h}\left[A_{k}(h)\right] .
$$

Substituting the Matrix Riccati equation for $A_{k}(h)$ in equation (8) into the above equation, we obtain

$$
\frac{d}{d h} G_{k}(h)-\frac{d}{d h}\left[F_{k}(h)\right] A_{k}(h)=G_{k}(h) \Phi_{k} F_{k}(h) \Gamma^{*}+\left[F_{k}(h)\left(\Phi_{k}^{\prime}+2 \Theta \gamma \rho_{k}^{\prime}\right)+2 G_{k}(h)\right] A_{k}(h) .
$$

Hence, we can construct a system of $(2 n)$ linear equations given by

$$
\begin{aligned}
\frac{d}{d h} G_{k}(h) & =F_{k}(h) \Gamma^{*}+G_{k}(h) \Phi_{k}, \\
\frac{d}{d h} F_{k}(h) & =-F_{k}(h)\left(\Phi_{k}^{\prime}+2 \Theta \gamma \rho_{k}^{\prime}\right)-2 G_{k}(h),
\end{aligned}
$$

with initial conditions $G_{k}(0)=O$ and $F_{k}(0)=I_{n}$. The above equations can be written as follows:

$$
\frac{d}{d h}\left(G_{k}(h) F_{k}(h)\right)=\left(\begin{array}{ll}
G_{k}(h) & \left.F_{k}(h)\right)
\end{array}\right)\left(\begin{array}{cc}
\Phi_{k} & -2 I_{n} \\
\Gamma^{*} & -\left(\Phi_{k}^{\prime}+2 \Theta \gamma \rho_{k}^{\prime}\right.
\end{array}\right) .
$$

Hence, we obtain the solution by matrix-exponentiation:

$$
\left(G_{k}(h) F_{k}(h)\right)=\left(\begin{array}{ll}
O & I_{n}
\end{array}\right) \operatorname{Exp} h\left(\begin{array}{cc}
\Phi_{k} & -2 I_{n} \\
\Gamma^{*} & -\left(\Phi_{k}^{\prime}+2 \Theta \gamma \rho_{k}^{\prime}\right.
\end{array}\right) .
$$

Using the definition of $N_{i j, k}(h) i, j=1,2$ in equation (9), we have $\left(G_{k}(h) \quad F_{k}(h)\right)=\left(N_{21, k}(h) N_{22, k}(h)\right)$, and $A_{k}(h)=\left[N_{22, k}(h)\right]^{-1} N_{21, k}(h)$. Now we turn to the ordinary differential equation for $c(h)$. Multiplying $F_{k}(h)$ before $\frac{d}{d h} F_{k}(h)$ above, we have

$$
\left[F_{k}(h)\right]^{-1} \frac{d}{d h} F_{k}(h)=-\left(\Phi_{k}^{\prime}+2 \Theta \gamma \rho_{k}^{\prime}\right)-2 A_{k}(h) .
$$


We can remove $A_{k}(h)$ from the ODE of $c(h)$, in order to give

$$
\frac{d}{d h} c(h)=-\frac{1}{2} \sum_{k=1}^{K} \nu_{k} \operatorname{tr}\left(\left[F_{k}(h)\right]^{-1} \frac{d}{d h} F_{k}(h)+\Phi_{k}^{\prime}+2 \Theta \gamma \rho_{k}^{\prime}\right)+\gamma^{\prime} \mu .
$$

Now we can integrate the last equation and obtain the solution of $c(h)$ given by equation (9).

\section{A.2 Proof of Proposition 2}

We can handle the conditional Laplace transform of the covariance process:

$$
\Psi_{\Xi, t}^{\dagger}(h)=E_{t}\left[\exp \left(\operatorname{tr}\left\{\Xi \Omega_{t+h}\right\}\right)\right]=\exp \left[\sum_{k=1}^{K} \operatorname{tr}\left(A_{k}^{\dagger}(h) V_{k t}\right)+b^{\dagger}(h)^{\prime} p_{t}+c^{\dagger}(h)\right],
$$

by replacing the corresponding boundary conditions and repeating the procedure in Appendix

A.1. Hence, we have

$$
\begin{aligned}
A_{k}^{\dagger}(h) & =\left[\Theta \Xi \Theta^{\prime} N_{12}(h)+N_{22, k}(h)\right]^{-1} \Theta \Xi \Theta^{\prime} N_{11, k}(h), \quad(k=1, \ldots, K) \\
b^{\dagger}(h) & =\mathbf{0}, \\
c^{\dagger}(h) & =-\frac{1}{2} \sum_{k=1}^{K} \nu_{k}\left[\log \operatorname{det}\left(N_{22, k}(h)\right)+h \operatorname{tr}\left(\Psi_{k}^{\prime}+2 \Theta \gamma \rho_{k}\right)\right],
\end{aligned}
$$

where

$$
\left(\begin{array}{cc}
N_{11, k}(h) & N_{12, k}(h) \\
N_{21, k}(h) & N_{22, k}(h)
\end{array}\right)=\operatorname{Exp} h\left(\begin{array}{cc}
\Psi_{k} & -2 I_{n} \\
O & -\Psi_{k}^{\prime}
\end{array}\right)
$$

It is straightforward to show that $N_{11, k}(h)=\operatorname{Exp}\left(\Psi_{k} h\right), N_{22, k}(h)=\operatorname{Exp}\left(-\Psi_{k}^{\prime} h\right), N_{21, k}(h)=O$ and $N_{12, k}(h)=-2 \Sigma(h) \operatorname{Exp}\left(-\Psi_{k}^{\prime} h\right)$, where $\Sigma(h)$ is defined by (11). By a property of matrixexponential transformation (for instance, see Chiu, Leonard and Tsui (1996)), we have $\log \operatorname{det}\left(N_{22, k}(h)\right)=\log \operatorname{det}\left(\operatorname{Exp}\left(-\Psi_{k}^{\prime} h\right)\right)=\operatorname{tr}\left(-\Psi_{k}^{\prime} h\right)$. Thus, we obtain

$$
\begin{aligned}
& A_{k}^{\dagger}(h)=\operatorname{Exp}\left(\Psi_{k}^{\prime} h\right)\left[I_{n}-2 \Theta \Xi \Theta^{\prime} \Sigma(h)\right]^{-1} \Theta \Xi \Theta^{\prime} \operatorname{Exp}\left(\Psi_{k} h\right), \\
& c^{\dagger}(h)=-\frac{1}{2} \sum_{k=1}^{K} \nu_{k} \log \operatorname{det}\left(I_{n}-2 \Theta \Xi \Theta^{\prime} \Sigma(h)\right) .
\end{aligned}
$$

Therefore, we have the results in (10). 


\section{A.3 Proof of Corollary 1}

In order to prove Corollary 1, we need to show that equation (13) is equivalent to the result obtained by Proposition 5 of Gourieroux (2006). We also need to show some properties of the matrix-exponential operator. Note that for any $n \times n$ matrix $A$, the spectral decomposition is given by $A=U L U^{-1}$, where $L$ is the diagonal matrix of eigenvalues such that $L=\operatorname{diag}\left\{\lambda_{1}, \ldots, \lambda_{m}\right\}$, and $U$ is the matrix of corresponding eigenvectors. Hence, we have $A^{i}=U L^{i} U^{-1}$, and we have the decomposition:

$$
\operatorname{Exp}(A)=U\left(\begin{array}{ccc}
\exp \left(\lambda_{1}\right) & & O \\
& \ddots & \\
O & & \exp \left(\lambda_{m}\right)
\end{array}\right) U^{-1} .
$$

(see Bellman (1970) and Chiu, Leonard and Tsui (1996)).

Lemma 1 For any square matrix $A(m \times m)$,

$$
\frac{d}{d s} \operatorname{Exp}(A s)=A \operatorname{Exp}(A s)
$$

Proof. It is straightforward to show this from the definition as:

$$
\begin{aligned}
\frac{d}{d s} \operatorname{Exp}(A s) & =\frac{d}{d s}\left\{I+\lim _{n \rightarrow \infty} \sum_{i=1}^{n} \frac{1}{i !} A^{i} s^{i}\right\}=\lim _{n \rightarrow \infty} \frac{d}{d s} \sum_{i=1}^{n} \frac{1}{i !} A^{i} s^{i} \\
& =\lim _{n \rightarrow \infty} A \sum_{i=1}^{n} \frac{1}{(i-1) !} A^{i-1} s^{i-1}=A \operatorname{Exp}(A s)
\end{aligned}
$$

Denote $G(h)=\operatorname{Exp}(\Phi h) C C^{\prime}[\operatorname{Exp}(P h i h)]^{\prime}$. Then, $\Omega(h)=\int_{0}^{h} G(s) d s$. Noting that $\frac{d G(s)}{d s}=\Phi G(s)+$ $G(s) \Phi^{\prime}$ by Lemma 1 , we have

$$
\operatorname{vec}(\Omega(h))=\left[\left(I_{m} \otimes \Phi\right)+\left(\Phi \otimes I_{m}\right)\right]^{-1} \operatorname{vec}\left(\operatorname{Exp}(\Phi h) C C^{\prime}[\operatorname{Exp}(\Phi h)]^{\prime}-C C^{\prime}\right),
$$

if $\Phi^{-1}$ exists. 


\section{References}

Aielli, G.P. (2006), "Consistent Estimation of Large Scale Dynamic Conditional Correlations", Working paper, Department of Statistics, University of Florence.

Asai, M., M. McAleer, and M.C. Medeiros (2012a), "Asymmetry and Long Memory in Volatility Modeling", Journal of Financial Econometrics, 10, 495-512.

Asai, M., M. McAleer, and M.C. Medeiros (2012b), "Estimation and Forecasting with Noisy Realized Volatility", Computational Statistics \& Data Analysis, 56, 217-230.

Asai, M., M. McAleer and J. Yu (2006), "Multivariate Stochastic Volatility: A Review", Econometric Reviews, 25, 145-175.

Barndorff-Nielsen, O.E., P.R. Hansen, A. Lunde, and N. Shephard (2011), "Multivariate Realised Kernels: Consistent Positive Semi-Definite Estimators of The Covariation of Equity Prices with Noise and Non-Synchronous Trading", Journal of Econometrics, 162, 149-169.

Barndorff-Nielsen, O. E., and N. Shephard (2002), "Econometric Analysis of Realized Volatility and Its Use in Estimating Stochastic Volatility Models", Journal of the Royal Statistical Society, Series B, 64, 253-280.

Bauwens, L., S. Laurent, and J.V.K. Rombouts (2006), "Multivariate GARCH: A Survey", Journal of Applied Econometrics, 21, 79-109

Bellman, R. (1970). Introduction to Matrix Analysis, New York: McGraw-Hill.

Bollerslev, T., and H. Zhou (2002), "Estimating Stochastic Volatility Diffusion Using Conditional Moments of Integrated Volatility", Journal of Econometrics, 109, 33-65.

Bru, M.-F. (1991), "Wishart Processes", Journal of Theoretical Probability, 4, 725-751.

Carr, P. and D.B. Madan (1999), "Option Valuation Using the Fast Fourier Transform", Journal of Computational Finance, 2, 61-73.

Chernov, M., A.R. Gallant, E. Ghysels, and G. Tauchen (2003), "Alternative Models for Stock Price Dynamics", Journal of Econometrics, 116, 225-257.

Chib, S., Y. Omori and M. Asai (2009), "Multivariate Stochastic Volatility", in: T.G. Andersen, R.A. Davis, J.-P. Kreiss and T. Mikosch (eds.), Handbook of Financial Time Series, SpringerVerlag, New York, 365-400. 
Chiu, T. Y. M., T. Leonard, K.-W. Tsui (1996), "The Matrix-Logarithmic Covariance Model", Journal of the American Statistical Association, 91, 198-210.

Drost, F.C. and B.J.M. Werker (1996), "Closing the GARCH GAP: Continuous Time GARCH Modeling", Journal of Econometrics, 74, 31-57.

Duan, J.-C. (1997), "The GARCH Option Pricing Model", Mathematical Finance, 5, 13-32.

Duffie, D., J. Pan, and K. Singleton (2000), "Transform Analysis and Asset Pricing for Affine Jump Diffusions", Econometrica, 68, 1343-1376.

Eberlein, E., K. Glau, and A. Papapantoleon (2010), "Analysis of Fourier Transform Valuation Formulas and Applications", Applied Mathematical Finance, 17, 211-240.

Engle, R.F. (2002), "Dynamic Conditional Correlation: A Simple Class of Multivariate Generalized Autoregressive Conditional Heteroskedasticity Models", Journal of Business \& Economic Statistics, 20, 339-350.

Fonseca, J., M. Grasselli, and C. Tebaldi (2008), "A Multifactor Volatility Heston Model”, Quantitative Finance, 8, 591-604.

Fornari, A. and A. Mele (1997), "Sign and Volatility Switching ARCH Models", Journal of Applied Econometrics, 12, 49-65.

Gourieroux, C. (2006), "Continuous Time Wishart Process for Stochastic Risk", Econometric Reviews, 25, 177-217.

Gourieroux, C., J. Jasiak, and R. Sufana (2009), "The Wishart Autoregressive Process of Multivariate Stochastic Volatility", Journal of Econometrics, 150, 167-181.

Gourieroux, C. and R. Sufana (2010), "Derivative Pricing with Wishart Multivariate Stochastic Volatility", Journal of Business \& Economic Statistics, 28, 438-451.

Hayashi, T. and N. Yoshida (2005), "On Covariance Estimation of Nonsynchronously Observed Diffusion Processes," Bernoulli, 11, 359-379.

Hafner, Laurent and Violante (2010), "The Diffusion Limit of Dynamic Conditional Correlation Models", Working paper, Université catholique de Louvain.

Heston, S.L. (1993), "A Closed-Form Solution for Options with Stochastic Volatility", Review of Financial Studies, 6, 327-343. 
Hull, J. and A. White (1987), "The Pricing of Options on Assets with Stochastic Volatility", Journal of Finance, 42, 281-300.

Malliavin, P. and M.E. Mancino (2009), "A Fourier Transform Method for Nonparametric Estimation of Multivariate Volatility", Annals of Statistics, 37, 1983-2010.

Melino, A. and S.M. Turnbull (1990), "Pricing foreign currency options with stochastic volatility", Journal of Econometrics, 45, 239-265.

Muhle-Karbe, J., O. Pfaffel, and R. Stelzer (2012), "Option Pricing in Multivariate Stochastic Volatility Models of OU Type", SIAM Journal on Financial Mathematics, 3, 66-94.

Nelson, D.B. (1990), "ARCH Models as Diffusion Approximations", Journal of Econometrics, $45,7-38$.

Nelson, D.B. (1996), "Asymptotic Filtering Theory for Multivariate ARCH Models", Journal of Econometrics, 71, 1-47.

Nelson, D.B. and D.P. Foster (1994), "Asymptotic Filtering Theory for Univariate ARCH Models", Econometrica, 62, 1-41.

Newey, W.K., and K.D. West (1987), "A Simple Positive Semi-Definite, Heteroskedasticity and Autocorrelation Consistent Covariance Matrix", Econometrica 55, 703-708.

Scott, L.O. (1987), "Option Pricing when the Variance Changes Randomly: Theory, Estimation and an Application", Journal of Financial and Quantitative Analysis, 22, 419-438.

Shephard, N. (2005), Stochastic Volatility: Selected Readings, Oxford: Oxford University Press.

Shoji, I., and T. Ozaki (1998), "A Statistical Method of Estimation and Simulation for Systems of Stochastic Differential Equations", Biometrika, 85, 240-243.

Tao, M., Y. Wang, Q. Yao, and J. Zou (2011), "Large Volatility Matrix Inference via Combining Low-Frequency and High-Frequency Approaches", Journal of the American Statistical Association, 106, 1025-1040.

Voev, V. and A. Lunde (2007), "Integrated Covariance Estimation Using Highfrequency Data in the Presence of Noise", Journal of Financial Econometrics, 5, 68-104.

Wang, Y., and J. Zou (2010), "Vast Volatility Matrix Estimation for High-Frequency Financial Data", Annals of Statistics, 38, 943-978. 
Zhang, L. (2008), "Estimating Covariation: Epps Effect, Microstructure Noise", Journal of Econometrics, 160, 33-47. 
Table 1: Monte Carlo Results for the GMM Estimator for Two-Factor Asymmetric WSV Model with Noise

\begin{tabular}{c|rcrc}
\hline \hline Para. & \multicolumn{1}{|c}{ True } & \multicolumn{1}{c}{ Mean } & Std.Dev. & RMSE \\
\hline$\mu_{1}$ & -0.0047 & -0.0212 & 0.00315 & 0.0169 \\
$\mu_{2}$ & -0.0013 & -0.0066 & 0.00130 & 0.0055 \\
$m_{11,1}$ & 0.8448 & 0.8432 & 0.00544 & 0.0057 \\
$m_{21,1}$ & -0.1867 & -0.1839 & 0.00586 & 0.0065 \\
$m_{22,1}$ & 0.1646 & 0.1710 & 0.00574 & 0.0085 \\
$m_{11,2}$ & 0.1805 & 0.1803 & 0.00389 & 0.0039 \\
$m_{21,2}$ & -0.0778 & -0.0774 & 0.00437 & 0.0044 \\
$m_{22,2}$ & 0.2429 & 0.2482 & 0.00618 & 0.0081 \\
$\theta_{11}$ & 0.0394 & 0.0388 & 0.00532 & 0.0054 \\
$\theta_{12}$ & 0.0070 & -0.0008 & 0.00549 & 0.0095 \\
$\theta_{22}$ & 0.0051 & -0.0073 & 0.00234 & 0.0126 \\
$\nu_{1}$ & 7.7153 & 7.7161 & 0.00375 & 0.0038 \\
$\phi_{11,1}$ & -7.1421 & -7.1417 & 0.00313 & 0.0032 \\
$\phi_{21,1}$ & -0.1148 & -0.1151 & 0.00299 & 0.0030 \\
$\phi_{12,1}$ & -0.0394 & -0.0395 & 0.00420 & 0.0042 \\
$\phi_{22,1}$ & -4.7369 & -4.7365 & 0.00650 & 0.0065 \\
$\rho_{1,1}$ & -0.3159 & -0.3158 & 0.00409 & 0.0041 \\
$\rho_{2,1}$ & -0.2855 & -0.2857 & 0.00415 & 0.0042 \\
$\nu_{2}$ & 2.8146 & 2.8151 & 0.01215 & 0.0122 \\
$\phi_{11,2}$ & -1.2305 & -1.2371 & 0.01705 & 0.0183 \\
$\phi_{21,2}$ & 0.8658 & 0.8648 & 0.00657 & 0.0066 \\
$\phi_{12,2}$ & -0.0967 & -0.1138 & 0.00975 & 0.0197 \\
$\phi_{22,2}$ & -0.0076 & -0.0269 & 0.01123 & 0.0223 \\
$\rho_{1,2}$ & -0.1095 & -0.1108 & 0.00612 & 0.0063 \\
$\rho_{2,2}$ & 0.1587 & 0.1604 & 0.00907 & 0.0092 \\
$\nu_{*}$ & 0.6882 & 2.4928 & 0.01888 & 0.0202 \\
\hline \hline
\end{tabular}


Table 2: GMM Estimates for One-Factor WSV Processes

\begin{tabular}{c|rcrrrrrr}
\hline \hline Para. & \multicolumn{2}{|c}{ WSV } & \multicolumn{2}{c}{ WSV-F } & \multicolumn{2}{c}{ WSV-L } & \multicolumn{2}{c}{ Asym. WSV } \\
\hline$\mu_{1}$ & -0.0005 & $(0.0002)$ & -0.0005 & $(0.0001)$ & -0.0004 & $(0.0002)$ & -0.0006 & $(0.0112)$ \\
$\mu_{2}$ & $6.17 \times 10^{-5}$ & $\left(5.06 \times 10^{-5}\right)$ & 0.0004 & $(0.0001)$ & $7.14 \times 10^{-5}$ & $(0.0001)$ & $-3.68 \times 10^{-5}$ & $(0.0019)$ \\
$m_{11,1}$ & & & 0.0186 & $(0.0039)$ & & & 0.2307 & $(0.0495)$ \\
$m_{21,1}$ & & & 0.0694 & $(0.0064)$ & & & -0.0270 & $(0.0283)$ \\
$m_{22,1}$ & & & 0.1131 & $(0.0372)$ & & & -0.0975 & $(0.0140)$ \\
$m_{11,2}$ & & & -0.1258 & $(0.0524)$ & & & -0.0277 & $(0.0168)$ \\
$m_{21,2}$ & & & -0.1646 & $(0.0039)$ & & & 0.2807 & $(0.0275)$ \\
$m_{22,2}$ & & & -0.2862 & $(0.0097)$ & & & 0.1422 & $(0.0765)$ \\
$\theta_{11}$ & 0.0234 & $(0.0044)$ & 0.0341 & $(0.0057)$ & 0.0324 & $(0.0053)$ & 0.0415 & $(0.0070)$ \\
$\theta_{12}$ & 0.0013 & $(0.0006)$ & -0.0025 & $(0.0054)$ & $6.57 \times 10^{-5}$ & $(0.0010)$ & 0.0022 & $(0.0071)$ \\
$\theta_{22}$ & -0.0114 & $(0.0021)$ & -0.0312 & $(0.0052)$ & -0.0303 & $(0.0051)$ & -0.0297 & $(0.0050)$ \\
$\nu_{1}$ & 17.004 & $(5.9687)$ & 2.0619 & $(0.3323)$ & 2.1981 & $(0.3206)$ & 2.1327 & $(0.3540)$ \\
$\phi_{11,1}$ & -3.0189 & $(1.1218)$ & -0.8373 & $(0.2688)$ & -0.7917 & $(0.2565)$ & -1.2414 & $(0.4102)$ \\
$\phi_{21,1}$ & -0.1031 & $(0.2372)$ & -0.3504 & $(0.5046)$ & -0.1273 & $(0.3385)$ & -0.0218 & $(0.6447)$ \\
$\phi_{12,1}$ & -0.0726 & $(0.0648)$ & -0.2729 & $(0.9217)$ & -0.2422 & $(0.9407)$ & -0.0869 & $(1.0963)$ \\
$\phi_{22,1}$ & -2.5587 & $(0.9563)$ & -2.1070 & $(0.7059)$ & -2.1170 & $(0.7084)$ & -1.9826 & $(0.6713)$ \\
$\rho_{1,1}$ & & & & & -0.4711 & $(0.2250)$ & -0.5232 & $(0.1502)$ \\
$\rho_{2,1}$ & & & & & -0.0461 & $(0.5941)$ & -0.0114 & $(0.0813)$ \\
$\nu_{*}$ & 0.1712 & $(0.0123)$ & 0.3139 & $(0.0354)$ & 0.2974 & $(0.0274)$ & 0.3052 & $(0.0329)$ \\
$J$-test & 25.015 & & 24.578 & & 24.378 & & 24.294 & $(7)$ \\
d.o.f. & $(15)$ & & $(9)$ & & $(13)$ & & 0.0010 & \\
$p$-value & 0.0497 & & 0.0035 & & 0.0278 & & & \\
\hline \hline
\end{tabular}

Note: Standard errors are in parentheses. ' $J$-test' is the GMM test of overidentifying restrictions. 
Table 3: Estimated Asymmetric Effects for One-Factor WSV Models

\begin{tabular}{c|rrrr}
\hline \hline Para. & WSV & WSV-F & WSV-L & Asym. WSV \\
\hline$E\left(\omega_{11, t}\right)$ & 0.001537 & 0.001506 & 0.001489 & 0.001485 \\
$E\left(\omega_{21, t}\right)$ & 0.000117 & 0.000105 & 0.000107 & 0.000104 \\
$E\left(\omega_{22, t}\right)$ & 0.000443 & 0.000486 & 0.000483 & 0.000481
\end{tabular}

Feedback Effect

\begin{tabular}{l|rrrr}
$E\left(\operatorname{tr}\left(M_{1} \Omega_{t}\right)\right)$ & 0 & $9.746 \times 10^{-5}$ & 0 & 0.00029003 \\
$E\left(\operatorname{tr}\left(M_{2} \Omega_{t}\right)\right)$ & 0 & -0.000363 & 0 & $8.575 \times 10^{-5}$
\end{tabular}

Leverage Effects from $u_{1 t}$

\begin{tabular}{l|lrrr}
$E\left(d \omega_{11, t} d u_{1 t}\right)$ & 0 & 0 & $-4.529 \times 10^{-5}$ & $-6.453 \times 10^{-5}$ \\
$E\left(d \omega_{21, t} d u_{1 t}\right)$ & 0 & 0 & $-0.039 \times 10^{-5}$ & $-0.712 \times 10^{-5}$ \\
$E\left(d \omega_{22, t} d u_{1 t}\right)$ & 0 & 0 & $0.016 \times 10^{-5}$ & $-0.019 \times 10^{-5}$
\end{tabular}

Leverage Effects from $u_{2 t}$

\begin{tabular}{l|rrrr}
$E\left(d \omega_{11, t} d u_{2 t}\right)$ & 0 & 0 & $-0.266 \times 10^{-5}$ & $-0.429 \times 10^{-5}$ \\
$E\left(d \omega_{21, t} d u_{2 t}\right)$ & 0 & 0 & $-1.918 \times 10^{-5}$ & $-2.378 \times 10^{-5}$ \\
$E\left(d \omega_{22, t} d u_{2 t}\right)$ & 0 & 0 & $0.078 \times 10^{-5}$ & $-0.106 \times 10^{-5}$ \\
\hline \hline
\end{tabular}

Note: We omitted $d t$ for leverage effects. 
Table 4: GMM Estimates for Two-Factor WSV Processes

\begin{tabular}{|c|c|c|c|c|c|c|c|c|}
\hline Para. & \multicolumn{2}{|c|}{ WSV } & \multicolumn{2}{|c|}{ WSV-F } & \multicolumn{2}{|c|}{ WSV-L } & \multicolumn{2}{|c|}{ Asym. WSV } \\
\hline$\mu_{1}$ & -0.0007 & $(0.0002)$ & -0.0013 & $(0.0002)$ & -0.0022 & $(0.0002)$ & -0.0047 & $(0.0006)$ \\
\hline$\mu_{2}$ & 0.0012 & $(0.0005)$ & 0.0005 & $(0.0001)$ & -0.0006 & $(0.0001)$ & -0.0013 & $(0.0003)$ \\
\hline$m_{11,1}$ & & & 0.0749 & $(0.0256)$ & & & 0.8448 & $(0.1870)$ \\
\hline$m_{21,1}$ & & & -0.1373 & $(0.0464)$ & & & -0.1867 & $(0.0734)$ \\
\hline$m_{22,1}$ & & & 0.0333 & $(0.0273)$ & & & 0.1646 & $(0.0470)$ \\
\hline$m_{11,2}$ & & & 0.0951 & $(0.0532)$ & & & 0.1805 & $(0.0503)$ \\
\hline$m_{21,2}$ & & & -0.0998 & $(0.0270)$ & & & -0.0778 & $(0.0066)$ \\
\hline$m_{22,2}$ & & & 0.0462 & $(0.0263)$ & & & 0.2429 & $(0.2668)$ \\
\hline$\theta_{11}$ & 0.0125 & $(0.0013)$ & 0.0123 & $(0.0005)$ & 0.0173 & $(0.0004)$ & 0.0394 & $(0.0011)$ \\
\hline$\theta_{12}$ & 0.0091 & $(0.0007)$ & 0.0087 & $(0.0003)$ & 0.0089 & $(0.0004)$ & 0.0070 & $(0.0004)$ \\
\hline$\theta_{22}$ & -0.0131 & $(0.0008)$ & -0.0128 & $(0.0004)$ & -0.0118 & $(0.0003)$ & 0.0051 & $(0.0002)$ \\
\hline$\nu_{1}$ & 5.7615 & $(1.7031)$ & 5.7753 & $(1.2572)$ & 5.6577 & $(2.4992)$ & 7.7153 & $(1.2193)$ \\
\hline$\phi_{11,1}$ & -5.2620 & $(0.6143)$ & -5.2497 & $(0.7034)$ & -5.3224 & $(1.1645)$ & -7.1421 & $(0.6422)$ \\
\hline$\phi_{21,1}$ & 0.0428 & $(0.0034)$ & 0.0360 & $(0.0111)$ & -0.0686 & $(0.0111)$ & -0.1148 & $(0.0231)$ \\
\hline$\phi_{12,1}$ & 0.0437 & $(0.0234)$ & 0.0365 & $(0.0069)$ & -0.0718 & $(0.0093)$ & -0.0394 & $(0.0073)$ \\
\hline$\phi_{22,1}$ & -5.0030 & $(0.7916)$ & -4.9987 & $(0.4301)$ & -5.0214 & $(1.2123)$ & -4.7369 & $(0.3698)$ \\
\hline$\rho_{1,1}$ & & & & & -0.2293 & $(0.0209)$ & -0.3159 & $(0.0236)$ \\
\hline$\rho_{2,1}$ & & & & & -0.2591 & $(0.0622)$ & -0.2855 & $(0.0207)$ \\
\hline$\nu_{2}$ & 4.4075 & $(0.3416)$ & 4.4533 & $(0.3251)$ & 3.9378 & $(0.3432)$ & 2.8146 & $(0.2341)$ \\
\hline$\phi_{11,2}$ & -0.7955 & $(0.0425)$ & -0.7348 & $(0.0137)$ & -0.5003 & $(0.0141)$ & -1.2305 & $(0.0741)$ \\
\hline$\phi_{21,2}$ & 0.7869 & $(0.0630)$ & 0.7314 & $(0.0203)$ & 0.1403 & $(0.0179)$ & 0.8658 & $(0.1434)$ \\
\hline$\phi_{12,2}$ & 0.9960 & $(0.0609)$ & 0.9404 & $(0.0197)$ & 0.4003 & $(0.0135)$ & -0.0967 & $(0.0067)$ \\
\hline$\phi_{22,2}$ & -1.2469 & $(0.0927)$ & -1.2034 & $(0.0308)$ & -0.3147 & $(0.0188)$ & -0.0076 & $(0.0050)$ \\
\hline$\rho_{1,2}$ & & & & & -0.4146 & $(0.0264)$ & -0.1095 & $(0.0166)$ \\
\hline$\rho_{2,2}$ & & & & & 0.1919 & $(0.0137)$ & 0.1587 & $(0.0767)$ \\
\hline$\nu_{*}$ & 1.5227 & $(0.0161)$ & 1.5359 & $(0.0162)$ & 0.6993 & $(0.0123)$ & 0.6882 & $(0.0124)$ \\
\hline$J$-test & 9.8073 & & 9.8073 & & 9.8071 & & 9.8062 & \\
\hline d.o.f. & $(16)$ & & $(10)$ & & (12) & & (6) & \\
\hline$p$-value & 0.8765 & & 0.4576 & & 0.6328 & & 0.1331 & \\
\hline
\end{tabular}

Note: Standard errors are in parentheses. ' $J$-test' is the GMM test of overidentifying restrictions. 
Table 5: Estimated Asymmetric Effects for Two-Factor WSV Models

\begin{tabular}{c|rrrr}
\hline \hline Para. & WSV & WSV-F & WSV-L & Asym. WSV \\
\hline$E\left(\omega_{11, t}\right)$ & 0.002332 & 0.002338 & 0.002718 & 0.002776 \\
$E\left(\omega_{21, t}\right)$ & 0.000182 & 0.000185 & 0.000208 & 0.000218 \\
$E\left(\omega_{22, t}\right)$ & 0.000434 & 0.000426 & 0.000815 & 0.000763
\end{tabular}

Feedback Effect

\begin{tabular}{l|llll}
$E\left(\operatorname{tr}\left(M_{1} \Omega_{t}\right)\right)$ & 0 & 0.000138 & 0 & 0.002389 \\
$E\left(\operatorname{tr}\left(M_{2} \Omega_{t}\right)\right)$ & 0 & 0.000205 & 0 & 0.000653
\end{tabular}

Leverage Effects from $u_{1 t}$

\begin{tabular}{l|llll}
$E\left(d \omega_{11, t} d u_{1 t}\right)$ & 0 & 0 & $-0.845 \times 10^{-5}$ & $-3.9434 \times 10^{-5}$ \\
$E\left(d \omega_{21, t} d u_{1 t}\right)$ & 0 & 0 & $-2.712 \times 10^{-5}$ & $-0.614 \times 10^{-5}$ \\
$E\left(d \omega_{22, t} d u_{1 t}\right)$ & 0 & 0 & $-0.344 \times 10^{-5}$ & $-0.025 \times 10^{-5}$
\end{tabular}

Leverage Effects from $u_{2 t}$

\begin{tabular}{l|rrrr}
$E\left(d \omega_{11, t} d u_{2 t}\right)$ & 0 & 0 & $-0.008 \times 10^{-5}$ & $-0.557 \times 10^{-5}$ \\
$E\left(d \omega_{21, t} d u_{2 t}\right)$ & 0 & 0 & $-0.537 \times 10^{-5}$ & $-0.556 \times 10^{-5}$ \\
$E\left(d \omega_{22, t} d u_{2 t}\right)$ & 0 & 0 & $-0.944 \times 10^{-5}$ & $0.029 \times 10^{-5}$ \\
\hline \hline
\end{tabular}

Note: We omitted $d t$ for leverage effects. 